\title{
Mobile Robot- Dynamic Model Controlling using Wavelet Network
}

\author{
Mohammed Kamil Hilfi \\ Department of Electrical Engineering \\ California State University, Fullerton
}

800 N. State College Blvd, Fullerton, CA 93834

\begin{abstract}
In this paper, mobile robot control system for dynamic model is implemented by using wavelet neural network and optimized by depending on PSO algorithm. The work is divided into two sections. In the $1^{\text {st }}$ section, the best structure of wavelet neural network controller is selected among different tested structures (by changing the number of neurons in hidden layer). In the $2^{\text {nd }}$ section, the best wavelet neural network controller is selected among different tested controllers by depending on the type of wavelet filter. The comparing is done by depending on the MSE values. The simulation is done by using MATLAB which reveals a good performance for the proposed control system.
\end{abstract}

\section{General Terms}

Mobile robot

\section{Keywords}

Mobile robot, dynamic model, wavelet neural network, PSO

\section{INTRODUCTION}

In recent years, mobile robots have been widely applied in lots of areas of different industrial applications because it can carry heavy mass with a very good balance such as loading transports. This is attracting the attention toward increasing the amount of research. Part of these research interest with the trajectory tracking problems.

The primary task for every mobile robot is to move along a defined trajectory [1].

There are two types of trajectory tracking controller which it can be used; reactive controller and predictive controller. Reactive controller is used with feedback control system that it generates mobile robot control signal by depending on the error signal (the difference between desired and actual signal). This means that the correction is done after the error happened, so it is impractical for using with high speed trajectory tracking in mobile robot application. While predictive controllers, it generates future information. The predicted state depends on the knowledge of the whole trajectory, and the model of mobile robot takes an advantage of actions that happened before the errors occur. This takes an ability for predictive trajectory tracking controller to be suitable for high speed trajectory tracking mobile in robot applications such as real time applications [2].

Differential drive mobile robot consists of a platform which is driven by two driving wheels, one in the left and the other in the right. These wheels mounted on the same axis with independent actuators. The movement of mobile robot is done by changing the relative angular velocities of driving wheels

The purpose of this paper is to propose a wavelet network based control scheme to solve the trajectory tracking problems

\author{
David Cheng \\ Department of Electrical Engineering \\ California State University, Fullerton \\ 800 N. State College Blvd, Fullerton, CA 93834
}

in a mobile robot dynamic model. The control system parameters will be optimized using PSO algorithm.

\section{WAVELET NEURAL NETWORK}

Most recently, the Wavelet Neural Network (WNN), has been utilizing in developing control system response. Wavelet neural network or simply wavenet, joins the wavelet transform theory with the basic concepts of neural networks to provide an alternative to neural network [3]. The usage of wavelet nodes led up to efficient networks which are optimal approximation for nonlinear functions. Wavelet neural network takes the advantages of high resolution nature of wavelets, learning, and feed forward nature of neural networks [4]. The combination of soft computing and wavelet theory has led to a number of new techniques which are wavelet networks and fuzzy-wavelet.

Wavelet networks (WNs) were presented in 1992 as a combination of artificial neural radial basis function (RBF) networks and wavelet decomposition. The advantage of the Wavelet Networks is that the wavelet coefficients are directly related data stream through the wavelet transform. In addition to that, the parameters of the wavelets in the WNs are subjected to be optimized [5].

Figure 1 shows the architecture of three layers wavelet neural network. The input layer consists of M nodes, the hidden layer consists of $\mathrm{N}$ nodes, and the output layer consists of $\mathrm{J}$ nodes. The impulse function of the hidden layer is wavelet basis function, and the impulse function of output layer is sigmoid function $[6,7]$. The input vector is $X=\left[\mathrm{x}_{1}, \mathrm{x}_{2}, \ldots \ldots, \mathrm{x}_{\mathrm{M}}\right]$, the actual output vector is $y=\left[y_{1}, y_{2} \ldots \ldots y_{J}\right]$, the expected output is $y_{d}=\left[y_{d_{1}}, y_{d_{2}}, \ldots \ldots, y_{d_{\mathrm{J}}}\right]$. The parameters $\mathrm{M}, \mathrm{N}, \mathrm{J}$ are the nodes number of the input layer, the hidden layer, and the output layer, respectively. The parameters $w_{i n, m}$ and $w_{o j, n}$ are the connecting weights between the input layer and the hidden layer, and between the hidden layer and the output layer, respectively.

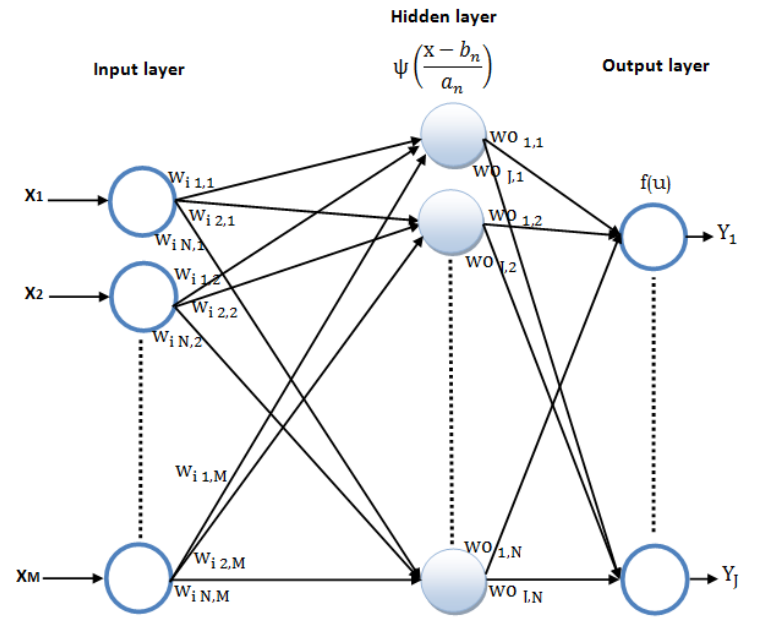

Fig 1: WNN Architecture [6]. 
The computation functions is as follows [8]:

$Y_{j}=f(u)=f\left[\sum_{n=1}^{N} w_{o j, n} \psi_{a n, b n}\left(\sum_{m=1}^{M} w_{i n, m} x_{m}\right)\right]$

$\mathrm{a}, \mathrm{b} \in \mathrm{R}, \quad \mathrm{a}>0$

$\mathrm{f}(u)=\frac{1}{1+\mathrm{e}^{-\mathrm{u}}}$

The sum of the output layer deviations is MSE,

MSE $=\frac{1}{\mathrm{~N}_{\mathrm{s}}} \sum_{i=1}^{\mathrm{N}_{\mathrm{s}}} \sum_{j=1}^{J}\left(\mathrm{y}_{\mathrm{d}_{\mathrm{j}}}(\mathrm{i})-\mathrm{y}_{\mathrm{j}}(\mathrm{i})\right)^{2}$

The wavelet network operation consists of two phases. In the first phase, the network architecture is determined for certain application. In the second phase, the parameter of the network are updated so that the approximation errors are minimized.

The wavelet neural network in Figure 1 is selected and used to determine the best motion controller for mobile robot which depends on the value of MSE. In the first test, the best architecture is found by changing the number of nodes in the hidden layer, and in the second test the number of wavelet functions are applied. The training algorithm which is used is PSO algorithm to select optimal values for wavelet neural network parameters (a, b, $w_{i n, m}$ and $\left.w_{o j, n}\right)$.

\section{THE PARTICLE SWARM OPTIMIZATION (PSO)}

Particle Swarm Optimization (PSO) is an evolutionary optimization technique which is introduced by Eberhart and Kennedy in 1995. One of the main streams of artificial life research is to examine how natural creatures behave as a swarm and reconfigure the swarm models inside a computer. School of fishes and swarm movement behavior, while they are searching for food [9].

The particle swarm optimization algorithm is used to find optimal parameters for the wavelet neural network as well as two PIDs parameters in the training phase, and that will minimize the error function in competitive time. The advantages of using particle swarm optimization (PSO) algorithm as compared with other techniques are that the PSO is computationally inexpensive, easily implemented, and does not require gradient information of an objective function, but only its values [10].

This modification can be represented by the concept of velocity. Velocity of each agent can be modified by the following equation [11].

$v_{i}^{k+1}=w v_{i}^{k}+c_{1} \operatorname{rand} 1\left(\right.$ pbest $\left._{i}-s_{i}^{k}\right)+c_{2} \operatorname{rand} 2\left(\right.$ gbest $\left._{i}-s_{i}^{k}\right)$

Using the above equation, a certain velocity, which gradually gets close to pbest and gbest can be calculated. The current position (searching point in the solution space) can be modified by the following equations.

$w=w_{\max }-\frac{\left(w_{\max }-w_{\min }\right)}{\text { iter }_{\max }}$

$s_{i}^{k+1}=s_{i}^{k}+v_{i}^{k+1}$
Where;

(w) is weight function, $\left(v_{i}^{k}\right)$ is velocity of agent $i$ at iteration $k$, $\left(c_{2}\right.$ and $c_{2}$ ) are weight factors, (gbest) is global best of the group, $\left(\right.$ pbest $\left._{\mathrm{i}}\right)$ is personal or local best of agent $\mathrm{i}$, $\left(\right.$ rand $_{1}$ and rand $\left._{2}\right)$ are random numbers between 0 and $1,\left(s_{i}^{k}\right)$ is current position of agent $\mathrm{i}$ at iteration $\mathrm{k},\left(\mathrm{w}_{\max }\right)$ is initial weight, $\mathrm{w}_{\min }$ is final weight, $\left(\right.$ iter $_{\max }$ ) is maximum number iteration.

The general flow chart of PSO is shown in Figure 2, and the step procedure is detailed below [12].

Step. 1 Generation of initial condition of each agent

Initial searching points $\left(s_{i}^{0}\right)$ and velocities $\left(v_{i}^{0}\right)$ of each agent are usually generated randomly within the allowable range. The current searching point is set to pbest for each agent. The best-evaluated value of pbest is set to gbest and the agent number with the best value is stored.

Step. 2 Evaluation of searching point of each agent

The objective function value is calculated for each agent. If the value is better than the current pbest of the agent, the pbest value is replaced by the current value. If the best value of pbest is better than the current gbest, gbest is replaced by the best value, and the agent number with the best value is stored.

Step. 3 Modification of each searching point: The current search point of each agent is changed using Equations (1), (2), and (3).

Step. 4 Checking the exit condition: If the current iteration number reaches the predetermined maximum iteration number, then exit. Otherwise, go to step 2.Figure2refer to the General flow chart of PSO.



Fig 2: The Flowchart of the Typical PSO [13]. 


\section{MOBILE ROBOT DYNAMIC MODEL}

The design of differential drive wheeled mobile robot is popular to use in designing the mobile robot. The advantage of this design is to separate the motion of two driving wheels which is making the mobile robot to be moved upright, curvy, or to turn on the position. The identical wheel speeds which depends on the translation between driving commands have to be accomplished by using a sophisticated software. The second advantage of this design is that the mechanical design of this type is very simple because the active wheels are in a stationary point and do not require to be turned as compared with the last design [14].

Dynamic modeling is the study of the motion in which forces and energies are modeled and included.

The dynamic model is derived from the physics laws that govern the several robot subsystems which is including the actuator dynamics (electric and mechanical characteristics of the motors), friction, and robot dynamics (movement equations). Dynamic modeling takes into account the forces acting on the vehicle [15]. This model can be constructed using the no-slip condition or allowing wheel slip. In either case, the acceleration of the car is considered. In dynamic modeling, the vehicle's dynamic properties such as mass, center of gravity, etc. enter into the equations. To derive this model, the nonholonomic constraints of the system are utilized as shown in figure 3 .

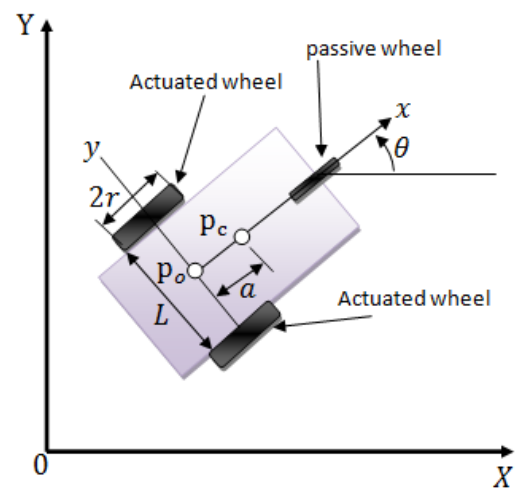

Fig 3: Two Wheel Driven Mobile Robot [16].

Dynamic equation of the wheeled mobile robot is described as the following [16].

$M(q) \dot{w}+C(q, q) w+D w=\tau$

with

$w=\left[w_{r} w_{l}\right], \quad \tau=\left[\tau_{v} \tau_{w}\right]$

$M(q)=\left[\begin{array}{ll}m_{11} & m_{12} \\ m_{12} & m_{11}\end{array}\right]$

$m=m_{c}+2 m_{w}$

$I=m_{c} a^{2}+2 m_{w} b^{2}+I_{c}+2 I_{m}$

$m_{11}=0.25 b^{-2} r^{2}\left(m b^{2}+I\right)+I_{w}$

$m_{12}=0.25 b^{-2} r^{2}\left(m b^{2}-I\right)$ $c=0.5 b^{-1} r^{2} m_{c} a$

$D=\left[\begin{array}{cc}d_{11} & 0 \\ 0 & d_{22}\end{array}\right]$

$c(q, \dot{q})=\left[\begin{array}{cc}0 & c \dot{\theta} \\ -c \dot{\theta} & 0\end{array}\right]$

$w=\dot{\theta}=\frac{d \theta}{d t}$

where

$w_{r}, w_{l}$ : are angular velocities of right and left wheel, respectively.

$m_{c}:$ is the mass of the body.

$m_{w}$ : is the mass of the wheel with a motor.

$I_{c}$ : is the moment of inertia of the body about the vertical axis through Pc (center of mass).

$I_{w}$ : is the moment of inertia of the wheel with a motor about the wheel axis.

$I_{m}:$ is the moment of inertia of the wheel with a motor about the wheel diameter.

$r:$ is the radius of the wheel.

$a:$ is the distance between the robot's center of mass and the center of the wheel axle.

$b:$ is the half distance between the two wheels (is half the width of the robot). $(\mathrm{L}=2 b)$

$d_{11}, d_{22}:$ are the damping coefficients.

$q=(\mathrm{x}, \mathrm{y}, \theta)$ : is the vector of generalized coordinates.

$W=\left(w_{r}, w_{l}\right):$ is the vector of velocities (right and left angular velocities respectively).

$\tau=(\tau 1, \tau 2)$ : is the vector of torques applied to the wheels of the robot (right and left wheel respectively).

$\tau_{1}$ : is the right motor torque, acting on the right wheel.

$\tau_{2}:$ is the left motor torque, acting on the left wheel.

$M(q)$ : is a $2 \times 2$ positive-definite inertia matrix.

$\mathrm{C}\left(q, \dot{q}^{\circ}\right)$ : is the vector of centripetal and Carioles forces.

$D$ : is a $2 \times 2$ diagonal positive-definite damping matrix (n vector gravitational torques).

To prepare for the control design in the next sections, the wheel velocities $w_{r}$ and $w_{l}$ will be converted to the linear $\mathrm{v}$ and angular $w$ velocities by the relationship.

$[v w]^{T}=R^{-1}\left[w_{r} w_{l}\right]^{T}$

$R^{-1}=\frac{1}{r}\left[\begin{array}{cc}1 & b \\ 1 & -b\end{array}\right]$ 
These are the equations that are used to build a model of the robot. These equations were used to simulate the robot in MATLAB Simulink. From the above equations,

$[\dot{v} \dot{w}]^{T}=-M^{-1}(q) D[v w]^{T}+M^{-1}(q)\left(-c(q, q)[v w]^{T}+\left[\tau_{r}, \tau_{l}\right]^{T}\right)$

Equation (4) can be rewritten as:

$\left[\begin{array}{c}\dot{v} \\ \dot{w}\end{array}\right]=-M^{-1}(q) D\left[\begin{array}{l}v \\ w\end{array}\right]+M^{-1}(q)\left[\begin{array}{c}-c \dot{\theta} w+\tau_{r} \\ c \dot{\theta} v+\tau_{l}\end{array}\right]$

The state, the manipulated, and the output variables for the robot will be defined as $[v w]^{T},\left[\tau_{r} \tau_{l}\right]^{T}$, and

$[v w]^{T}$, respectively. It is yielded the following state equations.

$\dot{x}=A x+B u$

$y=c x$

since

$A=-M^{-1}(q) D$

$B=M^{-1}(q)$

$C=\left[\begin{array}{ll}1 & 0 \\ 0 & 1\end{array}\right]$

\section{SIMULATION AND RESULTS}

The physical parameters for mobile robot dynamic model are taken from [16] as shown in Table 1.

Table 1. Physical Parameters of Mobile Robot Dynamic Model.

\begin{tabular}{|c|c|c|}
\hline Parameter & Value & Unit \\
\hline $\mathrm{a}$ & 0.3 & $\mathrm{~m}$ \\
\hline $\mathrm{b}$ & 0.75 & $\mathrm{~m}$ \\
\hline $\mathrm{c}$ & 0.135 & $\mathrm{Kg} \cdot \mathrm{m}^{2}$ \\
\hline$D_{11}$ & 10 & - \\
\hline$D_{22}$ & 10 & - \\
\hline$I_{c}$ & 15.625 & $\mathrm{Kg} \cdot \mathrm{m}^{2}$ \\
\hline$I_{m}$ & 0.0025 & $\mathrm{Kg} \cdot \mathrm{m}^{2}$ \\
\hline$I_{w}$ & 0.005 & $\mathrm{Kg} \cdot \mathrm{m}^{2}$ \\
\hline$m_{c}$ & 30 & $\mathrm{~kg}$ \\
\hline$m_{w}$ & 1 & $\mathrm{~kg}$ \\
\hline $\mathrm{r}$ & 0.15 & $\mathrm{~m}$ \\
\hline
\end{tabular}

Let the simulation setting is set with simulation period 30sec, and the following solver options: type fixed step size, solver type ode 4 runge-kutta, fundamental sample time $0.01 \mathrm{sec}$. Two paths used for finding the best structure which are linear and curvature with a linear velocity equal to $0.5 \mathrm{~m} / \mathrm{s}$. The PSO algorithm have the following setting values: no of birds is 50, Maximum iteration number is 250 , bird step is 250 , Wmax is 0.9 , Wmin is $0.25, c_{1}$ is 2.05 and $c_{2}$ is 2.05 .

The selection of the best motion controller for mobile robot depends on the measurements and comparing among these measurements. In this work paths (linear and curvature) are selected as shown in figure 4 and figure 5 .

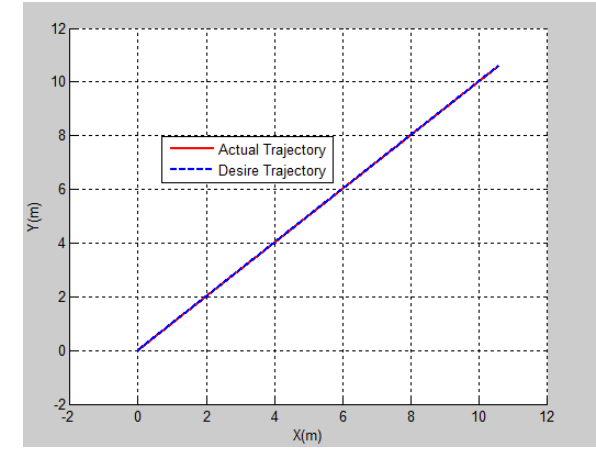

Fig 4: X-Y Response mobile robot control system for Linear Path.

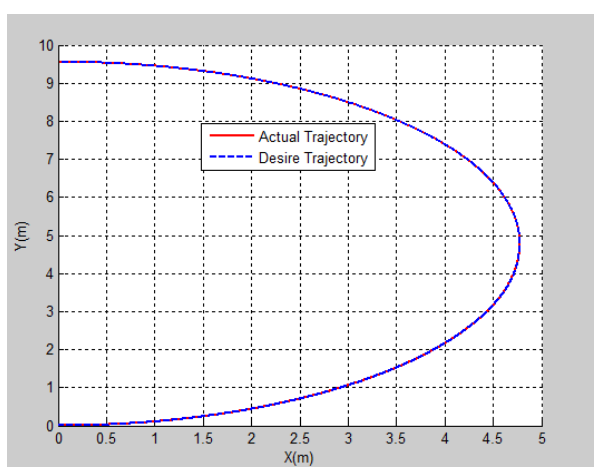

Fig 5: X-Y Response mobile robot control system for Curvature Path.

There are two testings, in the first test, morlet wavelet function and different number of neurons are used in hidden layer $(\mathrm{N}=10,20,30,40,50,100,150,200)$, and the comparing among these structures is done by depending on the value of MSE. As shown in table 2.

Table 2. Comparing Among Different WNN Structures

\begin{tabular}{|c|c|c|}
\hline $\mathrm{N}$ & MSE for Linear Path & MSE for Curvature Path \\
\hline 10 & 0.0139 & $1.0529 \mathrm{e}-004$ \\
\hline 20 & 0.0044 & $1.1294 \mathrm{e}-004$ \\
\hline 30 & 0.0014 & $1.0897 \mathrm{e}-004$ \\
\hline 40 & 0.0029 & $1.0257 \mathrm{e}-004$ \\
\hline 50 & 0.0035 & $1.0202 \mathrm{e}-004$ \\
\hline 100 & 0.0046 & $8.8505 \mathrm{e}-005$ \\
\hline 150 & 0.0012 & $8.7978 \mathrm{e}-005$ \\
\hline 200 & 0.0016 & $8.7857 \mathrm{e}-005$ \\
\hline
\end{tabular}

From the above result, the structure of $\mathrm{N}=30$ is the best structure, as compared with other structures because it has smallest values of MSE, while the controller of structure content $\mathrm{N}=150$ is inapplicable.

In the second test, different numbers of wavelet functions (Morlet, Polywog1, Shannon, Mexican hat) are used with fixed structure of $\mathrm{N}=30$, the comparing is done by depending on the smallest value of MSE. Results are detailed in the table 3. 
Table 3. Comparing Depends on Different Types of Wavelet Functions

\begin{tabular}{|c|c|c|}
\hline Wavelet Function & MSE for Linear Path & MSE for Curvature Path \\
\hline Morlet & 0.0014 & $1.0897 \mathrm{e}-004$ \\
\hline Polywog1 & 0.0292 & $5.2445 \mathrm{e}-004$ \\
\hline Shannon & 0.0145 & $5.2447 \mathrm{e}-004$ \\
\hline Mexican hat & 0.0094 & $5.2445 \mathrm{e}-004$ \\
\hline
\end{tabular}

From the above results, the structure of Morlet wavelet function and $\mathrm{N}=30$ is the best controller which is selected to be a motion controller for the mobile robot.

\section{CONCLUSION}

In this paper, mobile robot control system is implemented and tested by using matlab/simulink software package version 7.12.0 (R2011a), and the simulation results reveal that the method of using wavelet neural network as a motion controller is feasible because the wavelet neural network is succeeded to make the actual trajectory quickly approach to the desire trajectory in a short time. And the deviation between the actual and desire trajectories is very small. Also, the PSO algorithm is succeeded to optimize the wavelet neural network parameters by depending on the mean square error value.

\section{SUGGESTIONS FOR FUTURE WORK}

In the near future, we would propose two possible continuous studies. First is to measure the ability of the system for different masses with increment of $1 \mathrm{~kg}$ per procedure, i.e. $1 \mathrm{~kg}, 2 \mathrm{~kg}, \ldots . .5 \mathrm{~kg}$ etc. Second is to make the mobile robot autonomous by adding planning controller and connecting sensors to sense unknown environment such as laser range finder sensors.

\section{REFERENCES}

[1] P. ŠUSTER and A. JADLOVSKÁ, "TRACKING TRAJECTORY OF THE MOBILE ROBOT KHEPERA II USING APPROACHES OF ARTIFICIAL INTELLIGENCE," Acta Electrotechnica et Informatica, vol. 11, no. 1, pp. 38-43, 2011.

[2] J. R. Ferreira and A. P. Moreira, "Non-Linear Model Predictive Controller for Trajectory of an OmniDirectional Robot Using a Simplified Model," in 9th Portuguese Conference on Automatic Control, Controlo, Coimbra, Portugal , 2010

[3] W. Bellil, C. B. Amar and A. M. Alimi, "Comparison between Beta Wavelets Neural Networks, RBF Neural Networks and Polynomial Approximation for 1D, 2D Functions Approximation," PROCEEDINGS OF WORLD ACADEMY OF SCIENCE, ENGINEERING AND TECHNOLOGY VOLUME, pp. 102-107, 2006.

[4] P. Cristea, R. Tuduce and A. Cristea, "Time Series Prediction with Wavelet Neural Networks," 5th Seminar on Neural Network Applications in Electrical Engineering, pp. 5-10, 2000.

[5] M. M. M. S and D. P. Satyanarayana, "FPGA Implementation of Hybrid Architecture for Image Compression Optimized for Low Power and High Speed applications," International Journal of Scientific \& Engineering Research, vol. 4, no. 5, pp. 1931-1940, 2013.
[6] H. Chen, Y. Lu and L. Tu, "Fault Identification of Gearbox Degradation with Optimized Wavelet Neural Network," Shock and Vibration, vol. 20, no. 2, pp. 247262, 2013.

[7] Y. HUANG and S. YU, "Explosive Ordnance Disposal Robot Path Planning Based on Danger Model Immune Wavelet Neural Network vol. 3, no. 11.18," Advances in information Sciences and Service Sciences(AISS), 2011.

[8] T. Y. Abdalla and M. I. Hamzah, "Trajectory Tracking Control for Mobile Robot using Wavelet Network," International Journal of Computer Applications , vol. 74, no. 3, pp. 32-37, 2013.

[9] Q. Bai, "Analysis of Particle Swarm Optimization Algorithm," Computer and Information Science, vol. 3, no. 1, pp. 180-184, 2010.

[10] Z. A. Bashir and M. E. El-Hawary, "Applying Wavelets to Short-Term Load Forecasting Using PSO-Based Neural Networks," IEEE TRANSACTIONS ON POWER SYSTEMS, vol. 24, no. 1, pp. 20-27, 2009.

[11] J. Chao, W. Bo and H. Youmin, "Wavelet Neural Network Based on NARMAL2 Model for

Prediction of Thermal Characteristics in a Feed System," CHINESE JOURNAL OF MECHANICAL ENGINEERIN $G$, vol. 23, pp. 1-10, 2010 .

[12] Y. Bodyanskiy, I. Pliss and O. Vynokurova, "ADAPTIVE WAVELET-NEURO-FUZZY NETWORK IN THE FORECASTING AND EMULATION TASKS," International Journal "Information Theories \& Applications", vol. 15, pp. 47-54, 2008.

[13] S. Gholizadeh, "STRUCTURAL OPTIMIZATION FOR EARTHQUAKE LOADING WITH NONLINEAR RESPONSES BY SURROGATE MODELING BASED EVOLUTIONARY ALGORITHMS," ASIAN JOURNAL OF CIVIL ENGINEERING (BUILDING AND HOUSING), vol. 11, no. 1, pp. 25-42, 2010.

[14] R. Siegwart, I. R. Nourbakhsh and D. Scaramuzza, Introduction to Autonomous Mobile Robots, London, England: The MIT Press Cambridge, Massachusetts, 2004.

[15] E. P. Mendes and A. A. D. Medeiros, "IDENTIFICATION OF QUASI-LINEAR DYNAMIC MODEL WITH DEAD ZONE FOR MOBILE ROBOT WITH DIFFERENTIAL DRIVE," in Latin American Robotics Symposium and Intelligent Robotics Meeting, 2010.

[16] K. D. Do, Z.-P. Jiang and J. Pan, "A global outputfeedback controller for simultaneous tracking and stabilization of unicycle-type mobile robots," IEEE TRANSACTIONS ON ROBOTICS AND AUTOMATION, vol. 2, no. 3, pp. 589-594, 2004. 